Revista Brasileira de Meteorologia, v.25, n.3, 375 - 382, 2010

\title{
ASPECTOS ENERGÉTICOS DO DESENVOLVIMENTO DA CANA-DE-AÇÚCAR. PARTE 1: BALANÇO DE RADIAÇÃO E PARÂMETROS DERIVADOS
}

\author{
ROMISIO GERALDO BOUHID ANDRÉ ${ }^{1}$, JOSÉ CARLOS MENDONÇA ${ }^{2}$, VALDO DA SILVA \\ MARQUES $^{2}$, FRANCISCA MARIA ALVES PINHEIRO ${ }^{2}$, JOSÉ MARQUES ${ }^{3}$ \\ ${ }^{1}$ Instituto Nacional de Meteorologia (INMET/MAPA), Brasilia, DF \\ ${ }^{2}$ Laboratório de Meteorologia, Universidade Estadual do Norte Fluminense (LAMET/UENF), Macaé, RJ \\ ${ }^{3}$ Consultor, Rio de Janeiro, RJ
}

romisio.andre@inmet.gov.br, mendonça@uenf.br, valdo@lenep.uenf.br; francisca@lenep.uenf.br, marques1936@yahoo.com.br

Recebido Setembro 2008 - Aceito Março 2010

\begin{abstract}
RESUMO
Um experimento foi instalado no município de Campos dos Goytacazes, RJ, em uma área cultivada com cana-de-açúcar, onde foram medidos os dados de irradiância solar incidente (Rs $\downarrow$ ) e refletida pela superfície (Rs $\uparrow$ ) e do saldo de radiação (Rn). Foram analisados dados de quatro períodos sendo um no estádio de perfilamento (109 DAP), dois no estádio de desenvolvimento dos colmos (197 e 258 DAP) e um no estádio de maturação (294 DAP), bem como a variação diurna dos termos do balanço de radiação, a relação entre o saldo de radiação e o saldo de radiação global e também cálculos de alguns parâmetros derivados, tais como: albedo, coeficiente de troca de onda longa e coeficiente térmico. O albedo apresentou valores crescentes com o aumento do índice de área foliar (IAF) da cultura, com valor médio para o estádio de perfilamento de 0,24; para o estádio de desenvolvimento de colmos de 0,27 a 0,30 e para o estádio de maturação 0,31 . A média para todos os estádios foi de $0,28 \pm 0,029$. As regressões lineares entre o saldo de radiação e o saldo de ondas curtas apresentaram coeficientes de determinação de 0,99 . O coeficiente térmico $(\beta)$ variou de 0,061 a $-0,133$ e o de troca de onda longa $(\lambda)$ de 0,057 a 0,154 . Estes coeficientes mostraram ser ferramentas úteis nas transformações de energia na superfície e na atmosfera, por terem apresentado diferenças sensíveis nos diversos estádios fenológicos da cultura da cana-de- açúcar.
\end{abstract}

Palavras-chave: irradiância solar, albedo, coeficiente térmico, cana-de-açúcar

\begin{abstract}
ENERGY ASPECTS ON THE SUGAR CANE DEVELOPMENT. PART 1: RADIATION BALANCE AND DERIVED PARAMETERS

An experiment was installed in the Campos dos Goytacazes County, RJ, in a sugar cane crop area where solar incident irradiation (Rs $\downarrow$ ), outgoing radiation reflected by the surface (Rs $\uparrow$ ) and net radiation(Rn) were measured. Data from four periods were analyzed: one in the tillering stage (109 DAS), two in the development of sprouts (197 e 258 DAS) and one during maturation (294 DAS). The daily variation of each radiation balance element and the relation between net and global radiation were evaluated and parameters such as albedo, long wave exchange coefficient and heat coefficient were estimated. The albedo showed increasing values as the crop leaf area index (LAI) increases with mean value of 0.24 during tillering stage, 0.24 during the development of sprouts and 0.27 to 0.30 during maturation. The mean albedo for all stages was $0.28 \pm 0.03$. The linear regression between net radiation and net short wave radiation showed a determination coefficient of 0.99 . Heat coefficient $(\beta)$ varied from 0.061 to -0.133 and the long wave exchange coefficient $(\lambda)$ from -0.057 to 0.154 . These coefficients turn out to be useful tools in surface and atmosphere energy transformation because they showed significant differences during the phenological stages of sugar cane crop.
\end{abstract}

Keywords: solar irradiance, albedo, thermal coefficient, sugar cane 


\section{INTRODUÇÃO}

A região Norte Fluminense tem como uma de suas principais atividades econômicas a indústria sucroalcooleira, tendo gerado no ano de 2000 cerca de 175 milhões de reais e cerca de 15.000 empregos diretos e indiretos. Essa atividade, entretanto, nas últimas três décadas vem passando por um processo de declínio em função de sucessivos planos econômicos, desvalorização da moeda nacional em relação ao dólar, dívida em dólar assumidas pelas unidades produtivas na modernização das indústrias, fortes pressões competitivas impostas pelo mercado que exige produtividade e qualidade a custos cada vez menores, e falta de matéria prima (cana) devido ao déficit hídrico característico da região (Azevedo et al., 2002).

Segundo a Companhia Nacional de Abastecimento CONAB (2009) o Estado do Rio de Janeiro é o $11^{\circ}$ produtor de cana-de-açúcar do Brasil, tendo produzido na safra 2007/08 numa área de 100.399 ha, 3.831.652 toneladas do produto, sendo o município de Campos dos Goytacazes, situado no norte fluminense, o maior produtor de cana-de-açúcar do Estado.

A falta de informações sobre o ambiente de produção de cana-de-açúcar e sobre o impacto financeiro, que o setor canavieiro proporciona nessa região de produção, motivou os autores a realizar estudos sobre o balanço de radiação na cultura de cana-de-açúcar durante o seu desenvolvimento.

O clima é o fator que mais influencia na produtividade da cana-de-açúcar (Ide e Oliveira, 1986; Barbieri, 1993). No Brasil, devido à sua grande extensão territorial, encontram-se as mais variadas condições climáticas para o desenvolvimento da lavoura canavieira. Certas regiões possuem clima ideal sem restrição alguma, ao passo que outras apresentam restrições térmicas e/ou hídricas moderadas, que permitem a produção econômica da cultura sem exigir recursos e técnicas especiais. Entretanto, há aquelas onde há restrições limitantes e somente o cultivo de variedades selecionadas e o emprego de recursos extras podem corrigir as deficiências hídricas ou técnicas, viabilizando economicamente a atividade canavieira.

Alguns autores definem a temperatura como fator de maior importância para a maturação fisiológica da cana, porque, além de afetar a absorção de água e nutrientes por meio do fluxo transpiratório é um condicionante não controlável (Ometto, 1980; Ide e Banchi, 1984; Ide e Oliveira, 1986; Magalhães, 1987; Barbieri, 1993). A cana-de-açúcar desenvolve-se bem em regiões de clima quente. Temperaturas oscilando entre $26^{\circ} \mathrm{C}$ e $33{ }^{\circ} \mathrm{C}$ são favoráveis durante o estádio de desenvolvimento da cultura. Temperaturas inferiores a $21^{\circ} \mathrm{C}$ são favoráveis durante o estádio de maturação, pois reduzem a taxa de alongamento dos colmos e promovem o acúmulo de sacarose (Magalhães, 1987).

A umidade do solo é outro fator preponderante e varia em função do tipo de solo, do ciclo da cultura (cana planta ou soca), do estádio de desenvolvimento (ciclo fenológico), das condições climáticas e de outros fatores, como água disponível no solo e variedades cultivadas.

A distribuição da precipitação durante o ano é o fator mais importante para o desenvolvimento da cultura, pois pode haver deficiência hídrica nos diferentes estádios acarretando decréscimos na produtividade.

Para estudos de perda d'água para atmosfera em solos vegetados e comportamento de vários elementos meteorológicos, torna-se indispensável o conhecimento do balanço de radiação e suas variações, sendo necessárias medidas da irradiância solar incidente, da absorção dessa radiação de ondas curtas, da emissão da superfície e da atmosfera. Esse balanço deve ser complementado com determinações de coeficientes derivados do balanço de radiação, que traduzam as condições térmicas e hídricas da superfície e atmosfera vizinha, o efeito do sistema solo-atmosfera sobre as variáveis, componentes da produtividade agrícola da cultura.

O saldo de radiação à superfície consiste no balanço entre as entradas e saídas dos fluxos de radiação na interface superfície-atmosfera, e pode ser medido ou estimado com base nos componentes da irradiância solar de ondas curtas (incidente e refletida) e da radiação de ondas longas emitida pela superfície e pela atmosfera ( Fontana et al., 1991). Devido à sua importância para o desenvolvimento das culturas, o balanço de radiação tem sido objeto de vários trabalhos de pesquisa, como: Cunha et al. (1989) e André (1996), com a cultura do milho; André e Viswanadham (1983) e Fontana et al. (1991), com soja; André et al. (1988), com floresta; Pinheiro et al. (1994), com trigo; Alves e Azevedo (1997), com melão; e Liberato e Cardoso (2006), com análises das variações diárias dos termos do balanço de radiação em áreas de floresta e pastagem na Amazônia, para diferentes coberturas de nuvens.

A tendência dos valores de albedo é de aumentar com o desenvolvimento da cultura devido ao aumento da cobertura do solo, sendo evidente a relação entre o albedo e o IAF. Cunha et al. (1989) observaram que o albedo médio diário para a cultura do milho esteve diretamente relacionado com o IAF, variando de 0,19 a 0,24 .

Oliveira et al. (2007) avaliaram o desenvolvimento do sistema foliar em cultivares de cana-de-açúcar, em cana-planta e observaram IAF para as cultivares RB72454, RB855113 e RB855536 variando entre 1,5 e 2,0 aos 180 DAP em nove épocas de avaliação, na Estação Experimental de Paranavaí, PR.

Almeida et al. (2008) estudando o desenvolvimento vegetativo e produção de variedades de cana-de-açúcar em relação a disponibilidade hídrica e unidades térmicas no estado de Alagoas, estimaram o IAF entre 1,5 e 3,5 para as variedades RB92579, RB93509, RB931530 e SP791011 para a fase de perfilamento (DAP entre 150 e 160), sendo que as duas últimas variedades apresentaram IAF em torno de 2,5. 
André (1996), trabalhando com a cultura do milho, encontrou valores médios de albedo de 0,15 no estádio de desenvolvimento vegetativo, 0,17 no estádio de florescimento e 0,23 na maturação fisiológica, com valor médio para o ciclo da cultura de $0,16 \pm 0,04$. Para a cultura da soja, foram observados valores de albedo médio diário de 0,26 (Fontana et al.,1991) e 0,24 (André e Viswanadham, 1983), para cobertura completa do solo.

O presente trabalho é uma primeira parte de estudos experimentais mais abrangentes que estão sendo elaborados para a cultura de cana-de-açúcar, na região Norte Fluminense do Estado do Rio de Janeiro. Dessa forma, o objetivo deste trabalho foi analisar a magnitude dos termos do balanço de radiação e dos parâmetros derivados desse balanço, ao longo do ciclo de desenvolvimento da cana-de-açúcar, no município de Campos dos Goytacazes, RJ.

\section{MATERIAL E MÉTODOS}

\section{1 Área Experimental e Cultura Utilizada}

$\mathrm{O}$ experimento foi instalado em uma parcela de 5 ha, pertencentes à Usina Santa Cruz (latitude: $21^{\circ} 43^{\prime} 21,8^{\prime \prime}$; longitude: 4124'26,1'O'O; altitude:11 m). O solo do local é caracterizado como Cambissolo, com textura muito argilosa e relevo plano (Embrapa, 1999). Segundo a classificação de Köppen, o clima é do tipo Aw, ou seja, tropical úmido com verão chuvoso e inverno seco, sendo a temperatura do mês mais frio superior a $18^{\circ} \mathrm{C}$. As médias anuais de precipitação, temperatura e umidade relativa do ar são respectivamente, iguais a 1023 $\mathrm{mm}, 24^{\circ} \mathrm{C}$ e $77 \%$. O plantio foi realizado no dia 5 de agosto de 2005 e a colheita, em 17 de agosto de 2006, utilizando-se a variedade RB5536 no espaçamento de 1,40 m entre linhas, mantendo-se 13 gemas por metro. Foi utilizada cana planta com variedade precoce, com o objetivo de produzir cana sementes para plantio de outras áreas. No local foi instalada uma Estação Micrometeorológica Automática - EMA, para medidas das diferentes variáveis: temperatura e umidade do ar, velocidade do vento, saldo de radiação e irradiância solar (incidente e refletida).

A irradiância solar incidente (Rs $\downarrow$ ) e refletida pela superfície (Rs $\uparrow$ ) foram medidas por meio de 2 piranômetros LI-200X (Li-Cor, Lincoln, NE, USA), enquanto o saldo de radiação $(\mathrm{Rn})$ foi obtido por meio de um saldo radiômetro NR-Lite (Kipp e Zonen). Os sensores foram instalados a 2 $\mathrm{m}$ acima do dossel vegetativo, sendo ajustado sempre que necessário em função do crescimento da cultura, até o limite de 5,75 m, aos $276 \mathrm{DAP}$, quando a cana possuía 3,75 m de altura. A aquisição e o armazenamento dos dados foram realizados por meio de um coletor de dados (datalloger) modelo CR21X (Campbell Scientific INC.).
A precipitação pluviométrica do período do experimento está mostrada na Figura 1. Embora o total de chuva ter sido pouco maior que $600 \mathrm{~mm}$ e ocorreram períodos sem chuva, a cultura recebeu 3 irrigações de $30 \mathrm{~mm}$ cada na fase de estabelecimento, com intervalos de 40 dias entre elas. No entanto as plantas não apresentaram déficit hídrico em nenhum período.

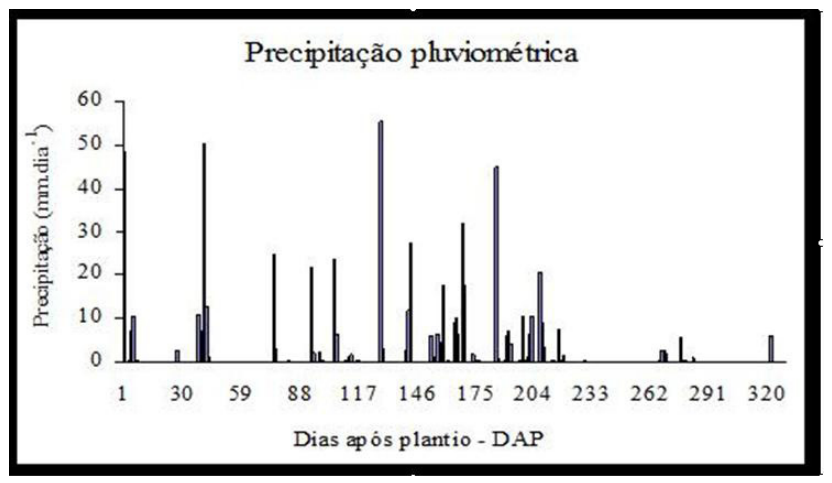

Figura 1 - Precipitação ocorrida durante o experimento.

\subsection{Balanço de Radiação}

O balanço de radiação na superfície do solo é dado de maneira aproximada por:

$$
R_{n}=R_{s} \downarrow-R_{s} \uparrow+L_{a}-L_{s}-(1-\varepsilon) L_{a}
$$

onde $R n$ é o saldo de radiação, $R s \downarrow$ a irradiância solar incidente, $R s \uparrow$ a irradiância solar refletida pela superfície, $L a$ a radiação atmosférica incidente à superfície, $L s$ a emissão efetiva da superfície e $\varepsilon$ a emissividade da superfície.

Foram analisados os seguintes parâmetros derivados do balanço de radiação, os quais são detalhadamente descritos em André e Viswanadham (1983) e André et al. (1988):

- $\alpha$ - albedo ou poder refletor da superfície, razão entre a irradiancia refletida $R_{s} \uparrow$ e a irradiancia solar incidente $\left(R_{s} \downarrow\right)$,

ou seja, $\alpha=\frac{R s \uparrow}{R s \downarrow}$

- a e b, respectivamente, coeficientes angular e linear da regressão entre o saldo de radiação $(\mathrm{Rn})$ e a diferença $\left(R_{s} \downarrow-R_{s} \uparrow\right)$ ou $\left.[1-\alpha) R_{s} \downarrow\right]$, saldo de radiação de ondas curta; Os parâmetros $\beta$ e $\lambda$ são obtidos com os coeficientes da regressão.

- $\lambda$ - coeficiente de troca de onda longa, relacionado com as propriedades térmicas do ar e definido por $\lambda=\mathrm{a}-1$. 
- $\beta$ - coeficiente térmico, relacionado com as propriedades térmicas da superfície considerada e definido por:

$$
\beta=\frac{1-a}{a}
$$

- A transmissividade da atmosfera foi calculada a partir da razão entre o fluxo integrado de radiancia solar incidente à superfície $\left(\mathrm{R}_{\mathrm{s}}\right)$ e aquele no topo da atmosfera $\left(\mathrm{R}_{\mathrm{a}}\right)$, a saber:

$$
N e b=\frac{R s \downarrow}{R a}
$$

- Os limites utilizados para Neb foram $(<0,30 ; 0,30-0,60$ e $>0,60)$, para dia nublado, parcialmente nublado e céu claro, respectivamente.

A radiação solar no topo da atmosfera foi obtida em função da latitude do local, da declinação solar e do ângulo horário, segundo a formulação dada por Vianello e Alves (1991):

$$
R a=S_{0}\left(\frac{D^{\prime}}{D}\right)^{2} \cos Z
$$

Onde $\mathrm{S}_{\mathrm{o}}$ é a constante solar, definida como $\left(\mathrm{S}_{\mathrm{o}}=1360\right.$ $\mathrm{W} \mathrm{m}^{-2}$ ), D é a distância Terra-Sol para um determinado dia do ano, D' é a distância média Terra-Sol e Z é o ângulo zenital, sendo $D \% D$ o fator de correção devido à excentricidade da órbita da Terra.

O saldo de radiação de ondas longas foi obtido por diferença entre o saldo de radiação $(\mathrm{Rn})$ e o saldo de ondas curtas, ou seja:

$$
\Delta L=R n-(1-\alpha) R s
$$

Para efeito de análise serão apresentados os resultados de quatro dias típicos um no estádio de perfilamento, dois no desenvolvimento de colmos e um, na maturação, com transmissividade atmosférica acima de $50 \%$ e totais diários dos diversos componentes do balanço de radiação. Os valores médios dos diversos estádios fenológicos, também serão apresentados. Todos os parâmetros derivados do balanço de radiação foram estimados para o período diurno, ou seja, para Rs $>0$.

As fases fenológicas consideradas foram: Perfilamento, do inicio até o DAP110 (DJ 329), de desenvolvimento dos colmos, entre os DAP 111 e 275(DJ330 a DJ 128) e a fase de maturação dos colmos do DAP 276 até 375(DJ 129 a DJ228).

\section{RESULTADOS E DISCUSSÃO}

Os resultados obtidos nos quatro dias característicos mostraram algumas semelhanças nos parâmetros derivados, que podem ser destacadas inicialmente. Por exemplo, o albedo mostrou valores altos nas primeiras horas da manhã e ao entardecer e menores valores no meio do dia. Estes resultados confirmam a dependência do albedo em função do ângulo de elevação solar; o valor mínimo por volta do meio-dia é atribuído ao menor ângulo de incidência dos raios solares, causando maior penetração e retenção da radiação no interior da comunidade vegetal. $\mathrm{O}$ saldo de ondas longas mostrou pequena variação durante o dia.

O dia 327/2005, com transmissividade atmosférica de 0,51 , foi selecionado para caracterizar o estádio de perfilamento, tendo apresentado os seguintes valores das variáveis atmosféricas: temperatura máxima $29,61{ }^{\circ} \mathrm{C}$, temperatura mínima $22,46{ }^{\circ} \mathrm{C}$, irradiância solar global 21,21 MJ $\mathrm{m}^{-2}$ e saldo de radiação $13,84 \mathrm{MJ} \mathrm{m}^{-2}$. A cultura se encontrava no estádio de perfilamento ( $\mathrm{DAP}=109)$, apresentando um índice de área foliar (IAF) entre 2,0 e 3,0 e as plantas apresentavam altura média de $1,2 \mathrm{~m}$.

Na Figura 2 é apresentada a variação diurna dos termos do balanço de radiação e do albedo para o dia especificado acima.

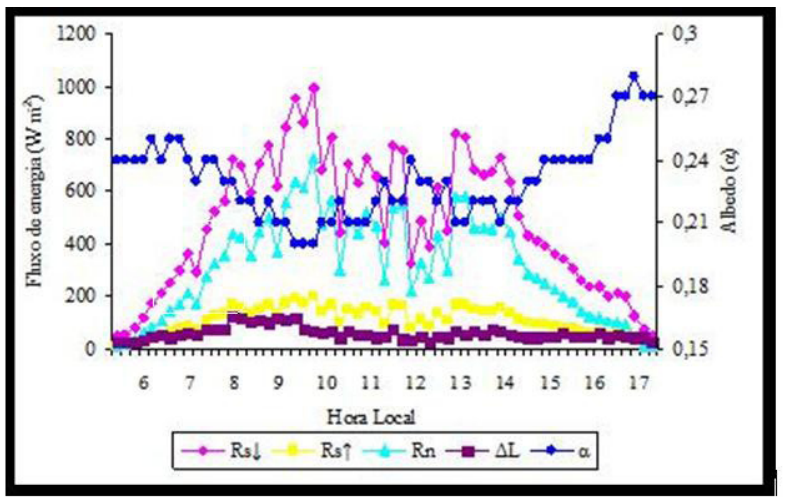

Figura 2 - Variação diurna dos componentes do balanço de radiação e do albedo para (DAP - 109), correspondente ao estádio de perfilamento da cultura da cana-de-açúcar: $\mathrm{Rs} \downarrow$ - irradiância solar incidente; Rs $\uparrow$ - irradiância refletida; $\mathrm{Rn}$ - saldo de radiação; $\Delta \mathrm{L}$ - saldo de ondas longas; $\alpha$ - albedo.

A irradiancia solar incidente e refletida, bem como o saldo de radiação, atingiram valores máximos de $994,00 \mathrm{~W} \mathrm{~m}^{-2}$, 202,5 e 723,86 $\mathrm{W} \mathrm{m}^{-2}$, respectivamente, medidos entre 10:00 e 10:30 horas local, conforme mostra a Figura 2. Os valores do albedo variaram entre 0,23 , por volta das 13 horas, cerca de 0,24 no início da manhã e 0,28 ao entardecer. $\mathrm{O}$ valor médio diurno do albedo para este dia foi $0,23 \pm 0,018$.

Os totais diurnos dos termos do balanço de radiação e os valores médios do albedo, representativos da fase de perfilamento estão apresentados na Figura 3.

A análise da Figura 3, para o estádio de perfilamento, mostra que o saldo de radiação esteve em fase com as 


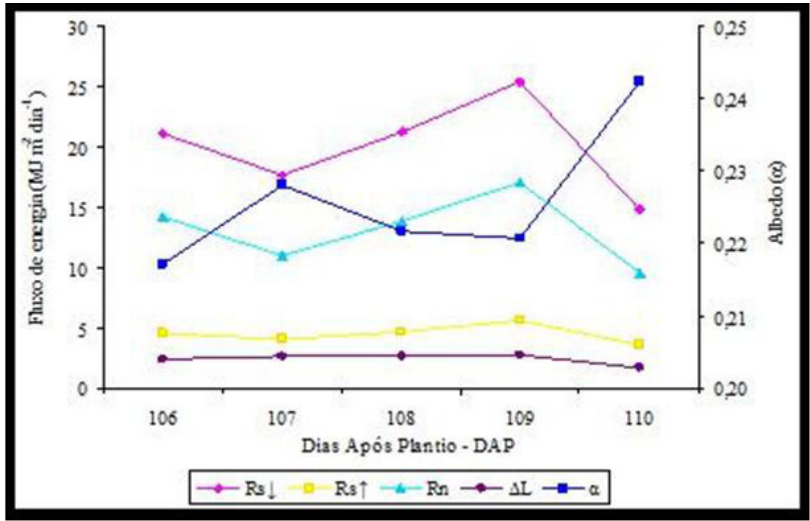

Figura 3 - Totais diurnos dos termos do balanço de radiação e valores médios do albedo, para o estádio de perfilamento da cana-de-açúcar. Rs $\downarrow$ - irradiância solar incidente; Rs $\uparrow$ irradiância refletida; $\mathrm{Rn}$ - saldo de radiação; $\Delta \mathrm{L}$ - saldo de ondas longas; $\alpha$ - albedo.

irradiâncias solar incidente e refletida. O saldo de radiação de onda longa mostrou ligeiro crescimento. $\mathrm{O}$ albedo médio, obtido pela média dos valores a cada 15 minutos, mostrou variações de 0,22 a 0,24 neste estádio.

O dia 050/2006, com transmissividade atmosférica de 0,66 , foi um dos dias selecionados para caracterizar o estádio inicial de desenvolvimento dos colmos, tendo apresentado os seguintes valores das variáveis atmosféricas: temperatura máxima $34,16{ }^{\circ} \mathrm{C}$, temperatura mínima $21,33{ }^{\circ} \mathrm{C}$, irradiância solar global $26,14 \mathrm{MJ} \mathrm{m}^{-2}$, saldo de radiação $17,26 \mathrm{MJ} \mathrm{m}^{-2}$. A cultura se encontrava no estádio inicial de desenvolvimento de colmos (DAP=197), apresentando um índice de área foliar (IAF) entre 6,0 e 7,0 e as plantas apresentavam altura média de 2,80 m.

A variação diurna dos termos do balanço de radiação e do albedo, representativos da fase inicial do desenvolvimento dos colmos estão apresentados na Figura 4.

A análise da Figura 4 mostra que Rs $\downarrow$ e Rn estão em fase durante todo o dia. As irradiâncias incidente e refletida, bem como, o saldo de radiação, atingiram valores máximos de $1093,00 \mathrm{~W} \mathrm{~m}^{-2}, 245,70 \mathrm{~W} \mathrm{~m}^{-2}$ e $828,00 \mathrm{~W} \mathrm{~m}^{-2}$, respectivamente. Esses valores foram medidos as 11:00 hora local. Os valores do albedo variaram entre 0,22 e 0,34 , com valor médio para esse dia foi de $0,28 \pm 0,051$.

Foi selecionado o dia 111/2006, com transmissividade atmosférica de 0,69 , para também caracterizar a fase de desenvolvimento dos colmos, uma vez que este é um estádio longo e importante para o desenvolvimento da cultura. Este dia apresentou os seguintes valores das variáveis atmosféricas: temperatura máxima de $28,96^{\circ} \mathrm{C}$, temperatura mínima de 16,35 ${ }^{\circ} \mathrm{C}$, irradiância solar global $20,36 \mathrm{MJ} \mathrm{m}^{-2}$ e saldo de radiação $14,35 \mathrm{MJ} \mathrm{m}^{-2}$.
A cultura encontrava-se na fase de desenvolvimento de colmos ( $\mathrm{DAP}=258$ ), apresentando um índice de área foliar (IAF) entre 7,0 e 8,0 e as plantas apresentavam altura média de 3,65 m.

A variação diurna dos termos do balanço de radiação e do albedo, representativos da fase de desenvolvimento dos colmos estão apresentados na Figura 5.

A irradiância solar incidente (Rs $\downarrow$ ), a refletida (Rs $\uparrow$ ) e o saldo de radiação $(\mathrm{Rn})$, apresentados na Figura 5 estão em fase durante todo o dia, atingindo valores máximos de $822,00 \mathrm{~W} \mathrm{~m}^{-2}$, $218,50 \mathrm{~W} \mathrm{~m}^{-2}, 635,00 \mathrm{~W} \mathrm{~m}^{-2}$, respectivamente. Esses valores máximos foram observados entre 11 e 13 horas (hora local). $\mathrm{O}$ albedo variou de 0,26 (12 h) a 0,41 no final da tarde, com valor médio de $0,31 \pm 0,043$.

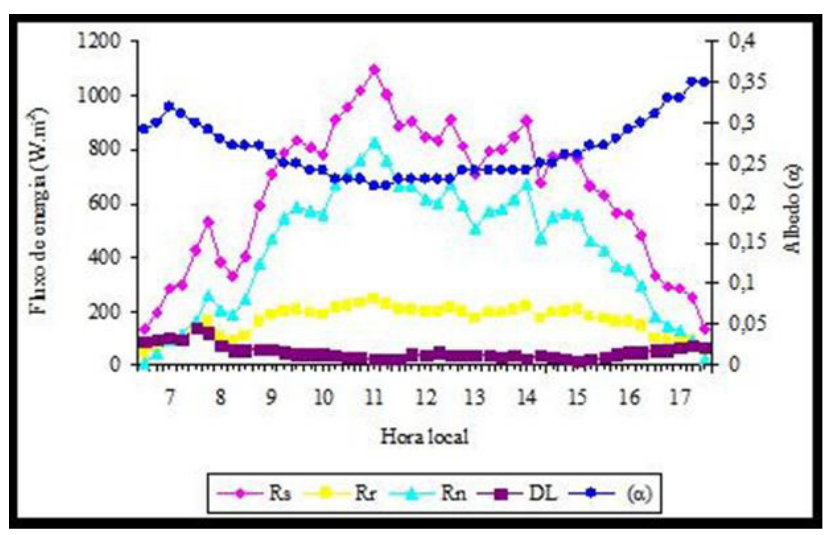

Figura 4 - Variação diurna dos componentes do balanço de radiação e do albedo (DAP=197), para o estádio inicial de desenvolvimento de colmos da cultura da cana-de-açúcar: Rs $\downarrow$ irradiância solar incidente; Rs $\uparrow$ - irradiância refletida; Rn - saldo de radiação; $\Delta \mathrm{L}$ - saldo de ondas longas; $\alpha$-albedo.

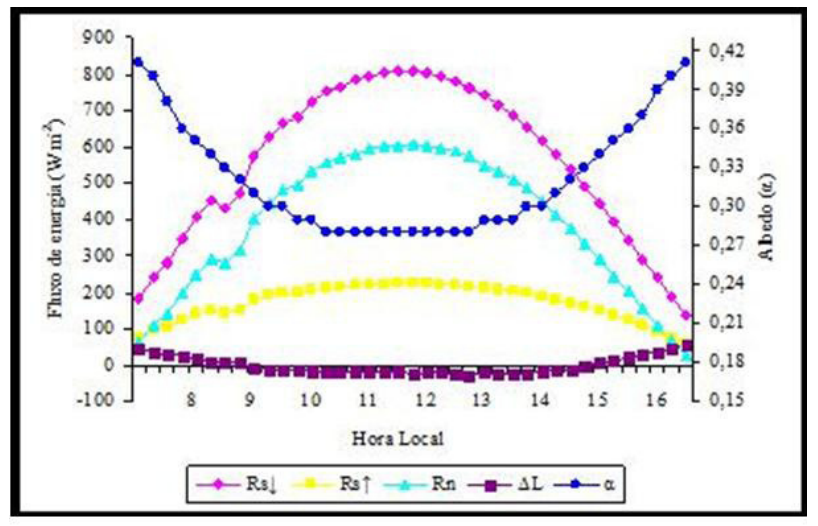

Figura 5 - Variação diurna dos componentes do balanço de radiação e do albedo( $(\mathrm{DAP}=258)$ para o estádio de desenvolvimento de colmos, da cultura da cana-de-açúcar: Rs $\downarrow$ - irradiância solar incidente; Rs $\uparrow$ - irradiância refletida; $\mathrm{Rn}$ - saldo de radiação; $\Delta \mathrm{L}$ - saldo de ondas longas; $\alpha$-albedo. 
Os totais diurnos dos termos do balanço de radiação e os valores médios do albedo, para fase de desenvolvimento dos colmos, estão apresentados na Figura 6.

A análise da Figura 6, para o estádio de desenvolvimento dos colmos, mostra que o saldo de radiação esteve em fase com as irradiâncias solar incidente e refletida. O saldo de radiação de onda longa mostrou valores bem pequenos. O albedo médio, obtido a cada 15 minutos, mostrou valores crescentes, variando de 0,23 a 0,30 . Isto se deve ao fechamento gradativo do dossel, com o desenvolvimento da cultura.

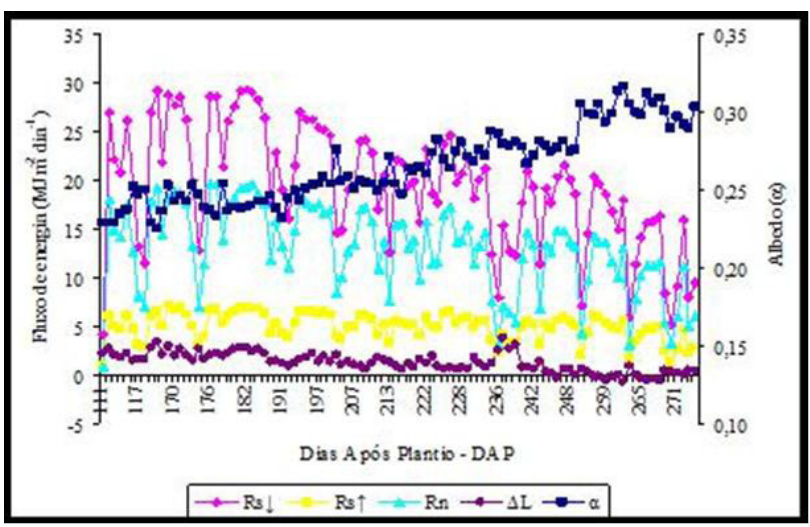

Figura 6 - Totais diurnos dos termos do balanço de radiação e valores médios do albedo, para o estádio de desenvolvimento de colmos da cultura da cana-de-açúcar: Rs $\downarrow$ - irradiância solar incidente; Rs $\uparrow$ - irradiância refletida; $\mathrm{Rn}$ - saldo de radiação; $\Delta \mathrm{L}$ - saldo de ondas longas; $\alpha$ - albedo.

O dia 147/2006, com transmissividade atmosférica de 0,63 , foi um dos dias selecionados para caracterizar o estádio de maturação, tendo apresentado os seguintes valores das variáveis atmosféricas: temperatura máxima de $28,12^{\circ} \mathrm{C}$, temperatura mínima de $13,94{ }^{\circ} \mathrm{C}$, irradiância solar $15,35 \mathrm{MJ} \mathrm{m}^{2}$, saldo de radiação10,15 $\mathrm{MJ} \mathrm{m}^{-2}$.

A cultura se encontrava no estádio de maturação ( $\mathrm{DAP}=294)$, apresentando um índice de área foliar (IAF) entre 8,0 e 9,0 e as plantas apresentavam altura média de $3,75 \mathrm{~m}$.

A Figura 7 mostra que as irradiâncias solar incidente $(R s \downarrow)$ e refletida (Rs $\uparrow$ ) e o saldo de radiação (Rn), estão em fase durante todo o dia, atingindo valores máximos de $659,00 \mathrm{~W} \mathrm{~m}^{-2}$ , 175,20 $\mathrm{W} \mathrm{m}^{-2}$ e $487,00 \mathrm{~W} \mathrm{~m}^{-2}$, respectivamente. Esses valores foram medidos entre as $12 \mathrm{e} 13 \mathrm{~h}$ (hora local). $\mathrm{O}$ albedo variou de 0,26 (entre 11 e $12 \mathrm{~h}$ ) a 0,37, com valor médio entre $0,31 \pm 0,034$.

Os totais diurnos dos termos do balanço de radiação e os valores médios do albedo, representativos da fase de maturação da cultura estão apresentados na Figura 8.

A análise desta Figura, para o estádio de maturação, mostra que o saldo de radiação esteve em fase com as

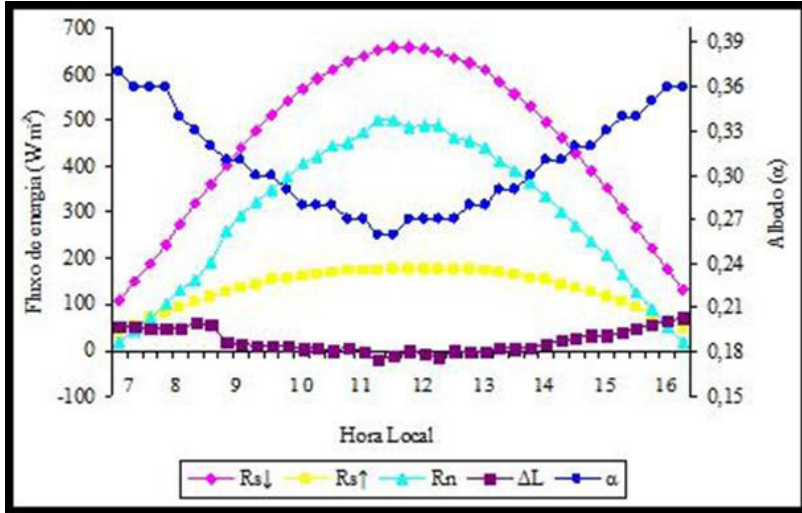

Figura 7 - Variação diurna dos componentes do balanço de radiação e do albedo $(\mathrm{DAP}=294)$, representando o estádio de maturação fisiológica da cultura da cana-de-açúcar (DV 2): $\mathrm{Rs} \downarrow$ - irradiância solar incidente; Rs $\uparrow$ - irradiância refletida; $\mathrm{Rn}$ - saldo de radiação; $\Delta \mathrm{L}$ - saldo de ondas longas; $\alpha$-albedo.

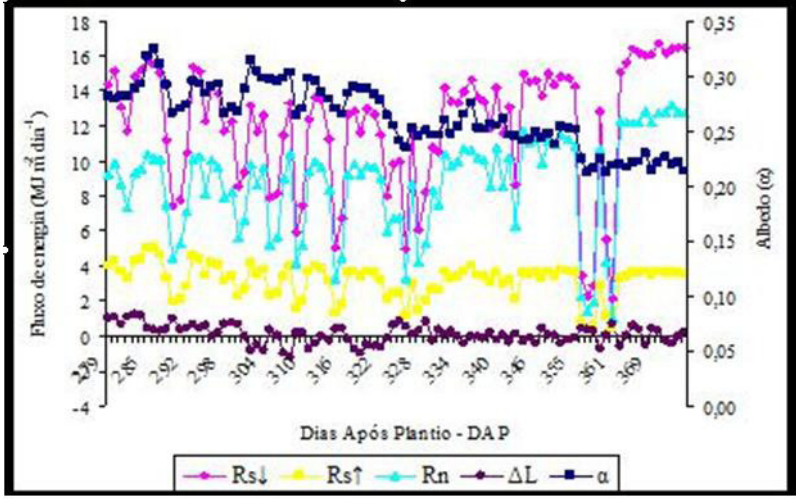

Figura 8 - Totais diurnos dos termos do balanço de radiação e valores médios do albedo, para o estádio de maturação fisiológica da cana-de-açúcar. Rs $\downarrow$ - irradiância solar incidente; $\mathrm{Rs} \uparrow$ - irradiância refletida; $\mathrm{Rn}$ - saldo de radiação; $\Delta \mathrm{L}$ - saldo de ondas longas; $\alpha$ - albedo.

irradiâncias solar incidente e refletida. O saldo de radiação de onda longa mostrou valores bem pequenos e, às vezes negativos. $\mathrm{O}$ albedo médio, obtido pela média dos valores a cada 15 minutos, mostrou valores decrescentes, variando de 0,28 a 0,21 . Isto se deve à perda de cobertura vegetal devido à senescência da parte aérea e conseqüente empalhamento das folhas.

Na Tabela 1 são apresentados os parâmetros derivados do balanço de radiação, nos estádios de perfilamento, desenvolvimento dos colmos e maturação, utilizando-se a equação de regressão entre Rn e [ (1- $\alpha) R s \downarrow$ ]

A análise da Tabela 1 mostra que o albedo aumentou com o desenvolvimento da cultura. Com relação aos coeficientes $\beta \mathrm{e}$ $\lambda$, nota-se que $\beta$ diminui com a elevação da cobertura vegetal, ocorrendo o contrário com $\lambda$. Fisicamente $\beta$ representa a parcela do saldo de radiação convertido em ondas longas. Assim, um 
aumento na radiação solar global, implica um aumento na emissão efetiva da superfície, o que provoca um aumento em $\beta$.

Os menores valores de $\beta$ (maiores valores de $\lambda$ ), no estádio de desenvolvimento vegetativo, mostraram que a maior parcela do saldo de radiação está sendo convertida em calor latente. Dessa forma, as magnitudes dos termos do balanço de radiação e dos parâmetros derivados, ao longo do ciclo de desenvolvimento da cana-de-açúcar serão usadas para os cálculos do balanço de energia e do fluxo de vapor d'água na segunda parte desse estudo.

\section{CONCLUSÕES}

Não houve mudanças no comportamento dos termos do balanço de radiação, quando se consideraram os diferentes estádios de desenvolvimento da cultura, embora os valores absolutos dos termos tenham sido diferentes.

$\mathrm{O}$ albedo mostrou valores crescentes com o aumento do IAF da cultura. Seu valor médio foi de 0,24 para o estádio de perfilamento, 0,27 para estádio inicial de desenvolvimento de colmos, 0,30 para o desenvolvimento de colmos e 0,31 para maturação. $\mathrm{O}$ valor médio do albedo para todo o ciclo da cultura foi $0,28 \pm 0,029$.

As regressões lineares entre Rn e (1- $\alpha)$ Rs $\downarrow$, ajustaram bem os dados para todos os dias considerados (representativo de cada estádio) e mostraram alta correlação.

O coeficiente térmico ( $\beta$ ) diminui com a elevação da cobertura vegetal, ocorrendo o contrário com o coeficiente de transformação em ondas londas $(\lambda)$.

Tabela 1 - Parâmetros relativos ao balanço de radiação para diferentes estádios vegetativos da cultura da cana-de-açúcar.

\begin{tabular}{|c|c|c|c|c|c|c|c|c|c|c|c|c|c|}
\hline Ano & DJ & DAP & Neb & $\alpha$ & DVP & $\mathbf{A}$ & $\mathbf{b}$ & $\mathbf{R}^{2}$ & $\beta$ & 2 & IAF & $\mathbf{h}$ & Estádios \\
\hline 2005 & 325 & 105 & 0,50 & 0,23 & 0,020 & 0,93 & $-25,70$ & 0,99 & 0,075 & $-0,070$ & & & Perfilamento \\
\hline 2005 & 526 & 106 & 0,42 & 0,23 & 0,017 & 0,93 & $-37,86$ & 0,98 & 0,075 & $-0,070$ & & & Perfilamento \\
\hline 2005 & 327 & 109 & 0,51 & 0,23 & 0,019 & 0,92 & $-31,60$ & 0,99 & 0,087 & $-0,080$ & $2,0-3,0$ & 1,2 & Perfilamento \\
\hline 2005 & 328 & 110 & 0,61 & 0,24 & 0,043 & 0,98 & $-54,81$ & 0,98 & 0,020 & $-0,020$ & & & Perfilamento \\
\hline 2005 & 329 & 111 & 0,35 & 0,25 & 0,010 & 0,91 & $-16,81$ & 0,99 & 0,099 & $-0,090$ & & & Perfilamento \\
\hline \multirow[t]{2}{*}{2005} & 331 & 113 & 0,64 & 0,25 & 0,038 & 0,99 & $-54,13$ & 0,99 & 0,010 & $-0,010$ & & & Perfilamento \\
\hline & & & & 0,24 & & & & & 0,061 & $-0,057$ & & & \\
\hline 2006 & 30 & 177 & 0,69 & 0,25 & 0,040 & 1,09 & $-105,9$ & 0,97 & $-0,083$ & 0,090 & & & D.Colmos \\
\hline 2006 & 31 & 178 & 0,69 & 0,25 & 0,040 & 1,07 & $-93,3$ & 0,98 & $-0,065$ & 0,070 & & & D.Colmos \\
\hline 2006 & 35 & 182 & 0,71 & 0,25 & 0,040 & 1,09 & $-122,1$ & 0,98 & $-0,083$ & 0,090 & & & D.Colmos \\
\hline 2006 & 48 & 195 & 0,68 & 0,26 & 0,036 & 1,08 & $-69,80$ & 0,99 & $-0,074$ & 0,080 & & & D.Colmos \\
\hline 2006 & 49 & 196 & 0,66 & 0,27 & 0,045 & 1,06 & $-63,43$ & 0,99 & $-0,057$ & 0,060 & & & D.Colmos \\
\hline 2006 & 50 & 197 & 0,66 & 0,28 & 0,051 & 1,06 & $-70,2$ & 0,99 & $-0,057$ & 0,060 & $5,0-6,0$ & 2,8 & D.Colmos \\
\hline 2006 & 51 & 198 & 0,64 & 0,28 & 0,050 & 1,04 & $-47,16$ & 0,99 & $-0,038$ & 0,040 & & & D.Colmos \\
\hline \multirow[t]{2}{*}{2006} & 52 & 199 & 0,64 & 0,28 & 0,041 & 1,08 & $-71,42$ & 0,99 & $-0,074$ & 0,080 & & & D.Colmos \\
\hline & & & & 0,27 & & & & & $-0,066$ & 0,071 & & & \\
\hline 2006 & 76 & 223 & 0,64 & 0,29 & 0,050 & 1,06 & $-49,91$ & 0,98 & $-0,057$ & 0,060 & & & D.Colmos \\
\hline 2006 & 79 & 226 & 0,67 & 0,29 & 0,050 & 1,07 & $-41,91$ & 0,99 & $-0,065$ & 0,070 & & & D.Colmos \\
\hline 2006 & 80 & 227 & 0,70 & 0,30 & 0,065 & 1,08 & $-50,76$ & 0,98 & $-0,074$ & 0,080 & & & D.Colmos \\
\hline 2006 & 83 & 230 & 0,64 & 0,29 & 0,050 & 1,06 & $-40,89$ & 0,98 & $-0,057$ & 0,060 & & & D.Colmos \\
\hline 2006 & 88 & 235 & 0,63 & 0,29 & 0,044 & 1,05 & $-40,74$ & 0,99 & $-0,048$ & 0,050 & & & D.Colmos \\
\hline 2006 & 100 & 247 & 0,64 & 0,29 & 0,033 & 1,12 & $-49,24$ & 0,99 & $-0,110$ & 0,124 & & & D.Colmos \\
\hline 2006 & 101 & 248 & 0,68 & 0,30 & 0,047 & 1,20 & $-98,84$ & 0,96 & $-0,163$ & 0,195 & & & D.Colmos \\
\hline 2006 & 111 & 258 & 0,69 & 0,31 & 0,043 & 1,17 & $-65,51$ & 0,99 & $-0,147$ & 0,173 & $7,0-8,0$ & 3,6 & \begin{tabular}{|l} 
D.Colmos \\
\end{tabular} \\
\hline 2006 & 112 & 259 & 0,67 & 0,32 & 0,043 & 1,14 & $-53,54$ & 0,99 & $-0,124$ & 0,142 & & & D.Colmos \\
\hline \multirow[t]{2}{*}{2006} & 113 & 260 & 0,64 & 0,31 & 0,038 & 1,17 & $-51,94$ & 0,99 & $-0,148$ & 0,174 & & & D.Colmos \\
\hline & & & & 0,30 & & & & & $-0,099$ & 0,113 & & & \\
\hline 2006 & 138 & 285 & 0,60 & 0,31 & 0,039 & 1,11 & $-69,97$ & 0,99 & $-0,099$ & 0,110 & & & Maturação \\
\hline 2006 & 139 & 286 & 0,63 & 0,33 & 0,029 & 1,20 & $-75,76$ & 0,99 & $-0,167$ & 0,200 & & & Maturação \\
\hline 2006 & 140 & 287 & 0,62 & 0,33 & 0,027 & 1,19 & $-73,81$ & 0,99 & $-0,160$ & 0,190 & & & Maturação \\
\hline 2006 & 141 & 288 & 0.60 & 0.33 & 0.034 & \begin{tabular}{|l|}
1.17 \\
\end{tabular} & -58.50 & 0.99 & -0.145 & 0.170 & & & Maturacão \\
\hline 2006 & 143 & 290 & 0,45 & 0,30 & 0,030 & 1,17 & $-47,47$ & 0,99 & $-0,145$ & 0,170 & & & Maturação \\
\hline 2006 & 146 & 293 & 0,43 & 0,28 & 0,018 & 1,06 & $-25,12$ & 0,99 & $-0,057$ & 0,060 & & & Maturação \\
\hline 2006 & 147 & 294 & 0,63 & 0,31 & 0,034 & 1,18 & $-76,97$ & 0,99 & $-0,153$ & 0,18 & $8,0-9,0$ & 3,8 & Maturação \\
\hline 2006 & 148 & 295 & 0,63 & 0,31 & 0,033 & 1,20 & $-77,01$ & 0,99 & & 0,20 & & & Maturação \\
\hline 2006 & 149 & 296 & 0,51 & 0,29 & 0,038 & 1,13 & $-52,65$ & 0,99 & $-0,115$ & 0,13 & & & Maturação \\
\hline 2006 & 150 & 297 & 0,59 & 0,30 & 0,022 & 1,13 & $-35,99$ & 0,99 & $-0,115$ & 0,13 & & & Maturação \\
\hline \multirow[t]{2}{*}{2006} & 151 & 298 & 0,58 & 0,30 & 0,025 & 1,17 & $-61,61$ & 0,99 & & 0,17 & & & Maturação \\
\hline & & & & 0,31 & & & & & $-0,133$ & 0,154 & & & \\
\hline
\end{tabular}

Dia escolhido para análise; DJ = Dia Juliano; DAP = dias após o plantio; Neb = nebulosidade; $\alpha=$ albedo; DVP = desvio padrão; $a$ = coeficiente angular; $b=$ coeficiente linear; $r 2$ = coeficiente de determinação; $\beta=$ coeficiente térmico; $\lambda=$ coeficiente de troca de onda longa; IAF $=$ índice de área foliar; $\mathrm{h}=$ altura da cultura (m). 


\section{REFERÊNCIAS BIBLIOGRÁFICAS}

ALMEIDA, A. C. DOS S., SOUZA, J. L., TEODORO, I., BARBOSA, G. V. S., FILHO, G. M., JÚNIOR, R. A. F., Desenvolvimento vegetativo e produção de variedades de cana-de-açúcar em relação a disponibilidade hídrica e unidades térmicas. Ciência Agrotecnica, Lavras, v. 32, n. 5, p. 1441-1448, set./out., 2008.

ALVES, A.V., AZEVEDO, P.V. Balanço de radiação num cultivo de melão. In: Congresso Brasileiro de Agrometeorologia, 10, 1997, Piracicaba. Anais...,Piracicaba: SBA, 1997. p.520-522. ANDRÉ, R.G.B., SILVA FILHO, V.P., MOLION, L.C.B., NOBRE, C.A. Balanço de radiação sobre a floresta amazônica (estações seca e úmida). Revista Brasileira de Meteorologia, v.3, p.269-274, 1988.

ANDRÉ, R.G.B., VISWANADHAM, Y. Radiation Balance of soybeans grown in Brazil. Agricultural and Forest Meteorology, Amsterdam, v.30, n.3, p.157-173, 1983. ANDRÉ, R.G.B. Aspectos energéticos e hídricos da cultura do milho (Zea mays) na região de Jaboticabal, SP. 1996. 96p. Tese (Livre - docência) Faculdade de Ciências Agrárias e Veterinárias de Jaboticabal-UNESP. Jaboticabal, SP, 1996.

AZEVEDO H. J., SILVA NETO, R. , CARVALHO, A. M. , VIANA, J. L. , MANSUR, A. F. U. Uma Análise da Cadeia Produtiva de Cana-de-Açúcar na Região Norte Fluminense. Observatório Sócio-Econômico da Região Norte Fluminense - Boletim Técnico n ${ }^{0}$ 6-51 p, 2002.

BARBIERI, V. Condicionamento climático da produtividade potencial da cana-de-açúcar (Saccharum spp.): um modelo matemático-fisiológico de estimativa.1993. 140p. Tese (Doutorado) - Escola Superior de Agricultura "Luiz de Queiroz”, Universidade de São Paulo. Piracicaba, SP. 1993.

CONAB - Companhia Nacional de Abastecimento. Safras. Capturado em 15 de julho 2009. On line. Disponível em http://www.conab.gov.br/conabweb/download/safra/1 cana de_acucar.pdf.

CUNHA,G.R., BERGAMACHI, H., BERLATO, M.A. Balanço de radiação em cultura de milho. In: Congresso Brasileiro de Agrometeorologia, 6, 1989, Maceió. Anais..., Maceió: SBA, 1989. p. 322-329.
EMBRAPA - Centro Nacional de Pesquisa de Solos. Sistema Brasileiro de Classificação de Solos - Brasília, DF. Embrapa Produção de Informação; Rio de Janeiro. 412 p, 1999.

FONTANA, D.C.; BERLATO, M.A. BERGAMASCHI, H. Balanço de radiação da soja em região subtropical do Brasil. Pesquisa Agropecuária Brasileira., v.26, n.3, p.411-8, 1991. IDE, B. Y.; BIANCHI, A. D. Influência do clima na produtividade da cana de açúcar In: Seminário de Tecnologia Agronômica, 2., Piracicaba, 1984. Anais... São Paulo: COPERSUCAR, 1984. p. 196-204

IDE, B. Y.; OLIVEIRA, M. A. DE. Efeito do clima na produção da cana-de-açúcar. In: Seminário de Tecnologia Agronômica, 3., Piracicaba, 1986. Anais... São Paulo: COPERSUCAR, 1986. p.573-583

LIBERATO, A.M.; CARDOSO, F.L. Estudo do balanço de radiação em áreas de floresta e pastagens na Amazônia. Acta Amazônica, no prelo, 2006.

MAGALHÃES, A. C. N. Ecofisiologia da cana-de-açúcar: aspectos do metabolismo do carbono na planta. In: Castro, P. R. C.; Ferreira, S. O.; Yamada, T. (Ed.). Ecofisiologia da Produção Agrícola. Piracicaba: Potafós, 1987. p.113-118. OLIVEIRA, R. A., DAROS E., ZAMBON J. L. C., WEBER. H., IDO O. T., BESPALHOK-FILHO J. C, J. C., ZUFFELLATO-RIBAS, K. C., SILVA, D. K. T. Área foliar em três cultivares de cana-de-açúcar e sua correlação com a produção de biomassa. Pesquisa Agropecuária Tropical. v. 2, n.37, p.71-76, 2007.

OMETTO, J. C. Parâmetros meteorológicos e a cultura da canade-açúcar. Piracicaba: ESALQ. 1980. 17p.

PINHEIRO, F.M.A.,PINHEIRO JR,H.S., ALVALÁ,R.C, GIELOW,R., SOUZA, J.R.S., MARQUES, J., VISWANADHAM,Y. Balanço de radiação em trigo de primavera crescido na Austrália. Boletim de Geografia Teorética,v.24, nos. 47-48, p.83-97, 1994.

VIANELlO, R.L., ALVES, A.R. Meteorologia básica e aplicações. Viçosa: Imprensa Universitária, UFV, 1991. 449P. 